\title{
Ramsey-nice families of graphs
}

\author{
Ron Aharoni * Noga Alon ${ }^{\dagger} \quad$ Michal Amir ${ }^{\ddagger}$ Penny Haxell ${ }^{\S}$ Dan Hefetz $₫$ \\ Zilin Jiang $\| \quad$ Gal Kronenberg ${ }^{* *} \quad$ Alon Naor ${ }^{\dagger \dagger}$
}

April 15, 2018

\begin{abstract}
For a finite family $\mathcal{F}$ of fixed graphs let $R_{k}(\mathcal{F})$ be the smallest integer $n$ for which every $k$-coloring of the edges of the complete graph $K_{n}$ yields a monochromatic copy of some $F \in \mathcal{F}$. We say that $\mathcal{F}$ is $k$-nice if for every graph $G$ with $\chi(G)=R_{k}(\mathcal{F})$ and for every $k$-coloring of $E(G)$ there exists a monochromatic copy of some $F \in \mathcal{F}$.

It is easy to see that if $\mathcal{F}$ contains no forest, then it is not $k$-nice for any $k$. It seems plausible to conjecture that a (weak) converse holds, namely, for any finite family of graphs $\mathcal{F}$ that contains at least one forest, and for all $k \geq k_{0}(\mathcal{F})$ (or at least for infinitely many values of $\left.k\right), \mathcal{F}$ is $k$-nice.

We prove several (modest) results in support of this conjecture, showing, in particular, that it holds for each of the three families consisting of two connected graphs with 3 edges each and observing that it holds for any family $\mathcal{F}$ containing a forest with at most 2 edges. We also study some related problems and disprove a conjecture by Aharoni, Charbit and Howard [1] regarding the size of matchings in regular 3-partite 3-uniform hypergraphs.
\end{abstract}

\section{Introduction}

In Ramsey theory, for a $k$-tuple $\left(H_{1}, \ldots, H_{k}\right)$ of fixed graphs, the Ramsey number $R\left(H_{1}, \ldots, H_{k}\right)$ is the smallest integer $n$ for which every coloring of $E\left(K_{n}\right)$ with the colors $1, \ldots, k$ yields a monochromatic copy (as a subgraph, not necessarily induced) of $H_{i}$ in the color $i$, for some $1 \leq i \leq k$. The special case where $H_{i}=K_{n_{i}}$ for every $1 \leq i \leq k$, is the well-studied Ramsey number $R\left(n_{1}, \ldots, n_{k}\right)$.

Instead of considering edge-colorings of cliques, one can extend the question to general graphs. Bialostocki and Gyárfás [9] asked for the smallest integer $n$ such that every graph $G$ with $\chi(G)=n$

\footnotetext{
*Department of Mathematics, Technion, Haifa 32000, Israel Email: raharoni@gmail.com

${ }^{\dagger}$ Sackler School of Mathematics and Blavatnik School of Computer Science, Tel Aviv University, Tel Aviv 6997801, Israel. Email: nogaa@tau.ac.il. Research supported in part by a BSF grant, an ISF grant and a GIF grant.

${ }^{\ddagger}$ School of Mathematical Sciences, Raymond and Beverly Sackler Faculty of Exact Sciences, Tel Aviv University, Tel Aviv, 6997801, Israel. Email: michalamir@mail.tau.ac.il.

${ }^{\S}$ Department of Combinatorics and Optimization, University of Waterloo, Waterloo, Ontario, Canada N2L 3 G1. Email: pehaxell@uwaterloo.ca. Partially supported by NSERC.

『Department of Computer Science, Ariel University, Ariel 40700, Israel. Email: danhe@ariel.ac.il

"Department of Mathematics, Technion - Israel Institute of Technology, Haifa, 32000, Israel. Email: jiangzilin@technion.ac.il.

** School of Mathematical Sciences, Raymond and Beverly Sackler Faculty of Exact Sciences, Tel Aviv University, Tel Aviv, 6997801, Israel. Email: galkrone@mail.tau.ac.il.

${ }^{\dagger \dagger}$ School of Mathematical Sciences, Raymond and Beverly Sackler Faculty of Exact Sciences, Tel Aviv University, Tel Aviv, 6997801, Israel. Email: alonnaor@post.tau.ac.il.
} 
(rather than just $K_{n}$ ) has the aforementioned Ramsey-type property. More specifically, they asked for which tuples $\left(H_{1}, \ldots, H_{k}\right)$ of fixed graphs we have the property that for every graph $G$ with $\chi(G)=$ $R\left(H_{1}, \ldots, H_{k}\right)$, and for every coloring of $E(G)$ with the colors $1, \ldots, k$, there exists a monochromatic copy (as a subgraph, not necessarily induced) of $H_{i}$ in the color $i$ for some $1 \leq i \leq k$. Such tuples are called Ramsey-nice. When $H_{1}=\ldots=H_{k}=H$ we say that $H$ is $k$-Ramsey-nice. For the remainder of this paper we abbreviate Ramsey-niceness simply to niceness ${ }^{1}$. Note that a tuple $\left(H_{1}, \ldots, H_{k}\right)$ is not nice if there exists an index $1 \leq i \leq k$ such that $H_{i}$ contains a cycle. Indeed, in his seminal paper [18] Erdős proved that for any two positive integers $\chi, g$ there exists a graph $G$ with $\chi(G)=\chi$ and girth greater than $g$. Therefore, if such an $i$ exists, we can find a graph with arbitrarily large chromatic number that does not contain $H_{i}$ as a subgraph and color all of its edges with the color $i$.

Extending a result of Cockayne and Lorimer [16] regarding the Ramsey number of matchings, Bialostocki and Gyárfás [9] proved that, for every positive integer $k$, the $k$-tuple $\left(M_{1}, \ldots, M_{k}\right)$ is nice, whenever $M_{i}$ is a matching (of any size $m_{i}$ ) for every $1 \leq i \leq k$. Garrison [21] observed that every star is $k$-nice for every $k$. Using a Turán-type argument, he proved that the path $P_{4}$ is $k$-nice for all $k$, except possibly for $k=3$, and that the pair $\left(P_{4}, P_{5}\right)$ is nice. Garrison also found a sufficient condition for a graph $H$ to be 2-nice, which he used to prove that $P_{5}, P_{6}$ and $P_{7}$ are all 2-nice.

In this paper, we study niceness of families of graphs rather than ordered tuples. For a family of graphs $\mathcal{F}$, let $R_{k}(\mathcal{F})$ denote the smallest integer $n$ for which in every $k$-coloring of $E\left(K_{n}\right)$ there exists a monochromatic copy of some $F \in \mathcal{F}$. We say that $\mathcal{F}$ is $k$-nice if for every graph $G$ with $\chi(G)=R_{k}(\mathcal{F})$ and for every $k$-coloring of $E(G)$, there is a monochromatic copy of some $F \in \mathcal{F}$. Note that if $H_{1}=\ldots=H_{k}=H$, then $R\left(H_{1}, \ldots, H_{k}\right)=R_{k}(\mathcal{F})$ where $\mathcal{F}=\{H\}$. If none of the elements in $\mathcal{F}$ is a forest, then by the same argument as in the tuple case, $\mathcal{F}$ is not $k$-nice for any $k$ (there exist graphs with arbitrarily large chromatic number containing no member of $\mathcal{F}$ as a subgraph). However, this argument does not apply if at least one $F \in \mathcal{F}$ is a forest as we do not associate each member of $\mathcal{F}$ with a specific color. More importantly, the number of colors $k$ used to color $E(G)$ does not depend on the size of the family $\mathcal{F}$.

In this paper we consider the following question.

Question 1.1. Is it true that for any finite family of graphs $\mathcal{F}$ that contains at least one forest, there exists a constant $k_{0}=k_{0}(\mathcal{F})$ such that $\mathcal{F}$ is $k$-nice for all $k \geq k_{0}$ ?

It is easy to see that the answer to this question is "yes" for any family containing a graph with at most 2 edges. We discuss this in greater detail in Section 6 . Our main focus here is families of connected graphs consisting of three edges each. Let $K_{3}$ be the triangle, let $P_{4}$ be the path on four vertices and let $S_{3}$ be the graph consisting of three edges with one common vertex (the star on four vertices). Note that these are the only connected graphs with three edges. Put $\mathcal{F}_{1}=\left\{K_{3}\right\}$, $\mathcal{F}_{2}=\left\{P_{4}\right\}, \mathcal{F}_{3}=\left\{S_{3}\right\}, \mathcal{F}_{4}=\left\{K_{3}, P_{4}\right\}, \mathcal{F}_{5}=\left\{K_{3}, S_{3}\right\}, \mathcal{F}_{6}=\left\{P_{4}, S_{3}\right\}, \mathcal{F}_{7}=\left\{K_{3}, P_{4}, S_{3}\right\}$.

Since the only member of $\mathcal{F}_{1}$ is a cycle, $\mathcal{F}_{1}$ is not $k$-nice for any $k$. As was already mentioned, it is shown in [21] that $\mathcal{F}_{2}$ is $k$-nice for every $k$, except possibly for $k=3$. This is proved using the known results that $R_{k}\left(P_{4}\right)=2 k+1$ for $k \equiv 0$ or $k \equiv 2(\bmod 3)($ but $k \neq 3), R_{k}\left(P_{4}\right)=2 k+2$ for $k \equiv 1(\bmod 3)$, and $R_{3}\left(P_{4}\right)=6($ see $[10,25])$. The only member of $\mathcal{F}_{3}$ is a star, hence the results in [21] imply that it is $k$-nice for all $k$. Moving on to the families that consist of two graphs, for the families $\mathcal{F}_{4}$ and $\mathcal{F}_{5}$ we can determine niceness for every $k$. However, the analysis for $\mathcal{F}_{6}$ is more complicated, involves some divisibility conditions and requires $k$ to be large enough in some cases.

\footnotetext{
${ }^{1}$ The author of [21] used the term good instead of nice. We change it to avoid ambiguity, as being $k$-good usually means something else in Ramsey Theory.
} 
For this purpose we define the following sets of integers (the specific value of $\Delta_{0}$ will be determined later in Definition 2.14).

Definition 1.2. Let

- $\mathcal{A}_{0}=\{n \in \mathbb{N} \mid n \equiv 0(\bmod 3)\} \backslash\{3,6,18,21,24,30,33,39,42,51,66\}$;

- $\mathcal{A}_{1}=\{n \in \mathbb{N} \mid n \equiv 1(\bmod 3)\}$;

- $\mathcal{A}_{2}=\left\{n \geq \Delta_{0} \mid n \equiv 2(\bmod 3)\right\} \cup\{2\}$;

- $\mathcal{A}=\mathcal{A}_{0} \cup \mathcal{A}_{1} \cup \mathcal{A}_{2}$.

Our main theorem is the following.

Theorem 1.3. Let $\mathcal{F}_{4}, \mathcal{F}_{5}, \mathcal{F}_{6}$ be as defined above. Then,

(1) $\mathcal{F}_{4}$ is $k$-nice for every $k$;

(2) $\mathcal{F}_{5}$ is $k$-nice for every $k \geq 2$, but not for $k=1$;

(3) $\mathcal{F}_{6}$ is $k$-nice for every $k \in \mathcal{A}$.

For the remaining family $\mathcal{F}_{7}$ we have the following result.

Theorem 1.4. $\mathcal{F}_{7}$ is $k$-nice for infinitely many integers $k$.

Even though Theorem 1.4 does not provide another example of a family for which the answer to Question 1.1 is affirmative, it does support the following weaker conjecture.

Conjecture 1.5. Any finite family of graphs $\mathcal{F}$ that contains at least one forest is $k$-nice for infinitely many integers $k$.

We can find support for this conjecture even if we do not limit the number of graphs in $\mathcal{F}$ or their sizes, as shown in the following theorem.

Theorem 1.6. Let $r$ be a positive integer, and let $\mathcal{F}$ be a family of graphs such that $K_{1, r+1} \in \mathcal{F}$, and all other $F \in \mathcal{F}$ contain at least one cycle. Then $\mathcal{F}$ is $k$-nice for infinitely many integers $k$.

The proofs of Theorems 1.3, 1.4 and 1.6 appear in Section 4.

The main ingredient in the proof of the last item of Theorem 1.3 is the answer to the following question: what is the maximum possible chromatic number of a graph obtained by taking the union of $r$ triangle factors on the same set of vertices (where the different factors are not necessarily edge disjoint)? A triangle factor is a graph in which every connected component is a triangle. We prove the following.

Theorem 1.7. The maximum possible chromatic number of a graph obtained by taking the union of $r$ triangle factors is:

(i) $2 r+1$ for $r \in \mathcal{A}_{1}$;

(ii) $2 r$ for $r \in \mathcal{A}_{0}$; 
(iii) $2 r-1$ for $r \in \mathcal{A}_{2}$.

This theorem settles the aforementioned question for all but a finite number of values of $r$. However, the set of integers for which we do not know the answer to that question, includes values as small as 3. Indeed, for $r=3$, the following question was suggested by Gyárfás [23].

Question 1.8. Suppose that $G$ is the union of three triangle factors on the same set of vertices. Is G 5-colorable?

We discuss this topic (including the proof of Theorem 1.7) in Section 3, and in addition show that the answer to Question 1.8 is "yes" if a conjecture by Molloy and Reed in [29] is affirmed. In fact, the affirmation of either their conjecture or a conjecture by Borodin and Kostochka in [12] will decrease the number of values of $r$ not covered by Theorem 1.7 to at most eleven.

The problems discussed in Section 3, and Question 1.8 in particular, are also related to problems on matchings in hypergraphs in the following sense. Let $\mathcal{G}_{n, r}$ be the family of all graphs obtained by taking the union of $r$ triangle factors, each containing $n$ triangles, on the same set of $3 n$ vertices. Let $\mathcal{H}_{n, r}$ be the family of all $r$-equipartite $r$-uniform 3-regular hypergraphs on $r n$ vertices. That is, every $\mathcal{H} \in \mathcal{H}_{n, r}$ has a vertex set $V(\mathcal{H})=V_{1} \cup \ldots \cup V_{r}$, each part satisfies $\left|V_{i}\right|=n$, every hyperedge has the form $\left\{v_{1}, \ldots, v_{r}\right\}$ where $v_{i} \in V_{i}$ for every $i$, and every vertex is contained in exactly 3 hyperedges (repeated hyperedges contribute with their multiplicities). The (simple) proof of the following lemma appears in the next section.

\section{Lemma 1.9.}

$$
\max _{G \in \mathcal{G}_{n, r}} \chi(G)=\max _{\mathcal{H} \in \mathcal{H}_{n, r}} \chi^{\prime}(\mathcal{H}) .
$$

Note that every color class in any proper edge coloring of a hypergraph is a matching. Therefore, by Lemma 1.9, a low chromatic number of members of $\mathcal{G}_{n, r}$ implies a low chromatic index of the corresponding members of $\mathcal{H}_{n, r}$, which, in turn, by the pigeonhole principle, implies the existence of a large matching in these hypergraphs. In particular, if the answer to Question 1.8 is "yes", and so $\chi(G) \leq 5$ for every $n$ and for every $G \in \mathcal{G}_{n, 3}$, then the following holds: In every 3-regular $n \times n \times n$, 3-partite 3-uniform hypergraph (we define an $n \times n \times n$ hypergraph to be a 3-equipartite 3-uniform hypergraph on $3 n$ vertices), there exists a matching of size at least $\left\lceil\frac{3}{5} n\right\rceil$. This is proved in [14] by Cavenagh, Kuhl and Wanless for such hypergraphs assuming they have no repeated edges.

One can consider a similar question in a more general setting: what is the largest matching guaranteed in any $d$-regular $r$-partite $r$-uniform hypergraph? Aharoni, Charbit and Howard conjectured the following for $r=3$.

Conjecture 1.10 (Conjecture 9.3 in [1]). In any d-regular $n \times n \times n$, 3-partite, 3-uniform hypergraph not containing repeated edges, there exists a matching of size at least $\left\lceil\frac{d-1}{d} n\right\rceil$.

We disprove this conjecture by a large margin in Section 5 by showing that, even for arbitrarily large $d$, there are such hypergraphs containing no matching of size larger than $2 n / 3$. A special case of a conjecture in [3], if true, implies that $2 n / 3$ is also a lower bound, even if the hypergraph is not 3-partite. It is also known (see, e.g., [4]), that if the hypergraph is linear, that is, contains no two edges that share more than one common vertex, then there is always a matching of size at least $\left(1-O\left(\frac{\log ^{3 / 2} d}{\sqrt{d}}\right)\right) n$. For large values of $r$, we show that there exist $r$-uniform $r$-partite $d$-regular hypergraphs on $n$ vertices, for arbitrarily large $d$, such that the largest matching covers only $(1+o(1)) \frac{n}{r}$ vertices, which is asymptotically tight. 


\section{Preliminaries and notation}

For every positive integer $k$ we use $[k]$ to denote the set $\{1,2, \ldots, k\}$. Our graph-theoretic notation is standard and follows that of [37]. In particular, we use the following.

For a graph $G=(V, E)$ let $\bar{G}=(V, \bar{E})$ denote the complement graph of $G$, that is, $\bar{E}=\{u v \mid u \neq$ $v \in V, u v \notin E\}$. For a set of vertices $U \subseteq V(G)$, the subgraph of $G$ induced by $U$ is denoted by $G[U]$. For a subset $U \subseteq V$, let $N_{G}(U)=\{v \in V \backslash U \mid \exists u \in U$ such that $u v \in E(G)\}$ denote the external neighborhood of $U$ in $G$. For a vertex $v \in V$ we abbreviate $N_{G}(\{v\})$ to $N_{G}(v)$ and let $d_{G}(v)=\left|N_{G}(v)\right|$ denote the degree of $v$ in $G$. The maximum degree and the minimum degree in $G$ are denoted by $\Delta(G)$ and $\delta(G)$, respectively. Often, when there is no risk of ambiguity, we omit the subscript $G$ in the above notation.

The size of a largest clique in $G$ is denoted by $\omega(G)$. For an integer $k$, the $k$-core of a graph $G$ is the (unique) maximal subgraph of $G$ in which all vertices have degree at least $k$. If no such subgraph exists we say that $G$ has an empty $k$-core.

A $k$-coloring of a graph $G$ is a function $f: V(G) \rightarrow[k]$. A coloring $f$ of $G$ is called proper if $f(v) \neq f(u)$ for every pair of adjacent vertices $u, v \in V(G)$. The graph $G$ is called $k$-colorable if there exists a proper $k$-coloring of $G$. The chromatic number of a graph $G$, denoted by $\chi(G)$, is the minimal $k$ for which $G$ is $k$-colorable. Similarly, a $k$-edge-coloring of $G$ is a function $f: E(G) \rightarrow[k]$, it is proper if $f\left(e_{1}\right) \neq f\left(e_{2}\right)$ for every pair of intersecting edges $e_{1}, e_{2}$, and the chromatic index of a graph $G$, denoted by $\chi^{\prime}(G)$, is the minimal $k$ for which there exists a proper $k$-edge-coloring of $G$. A graph $G$ is called $s$-critical if $\chi(G)=s$ and $\chi(H)<s$ for every proper subgraph $H$ of $G$.

A set of graphs $\left\{G_{1}, \ldots, G_{k}\right\}$ is a covering of a graph $G$ if $E(G) \subseteq \bigcup_{i=1}^{k} E\left(G_{i}\right)$. Such a set is called a decomposition of $G$ if, in addition, $E\left(G_{i}\right) \subseteq E(G)$ for every $1 \leq i \leq k$, and all the graphs in this set are pairwise edge-disjoint. For a fixed graph $H$ on $h$ vertices, and for an $n$-vertex graph $G$ with $n$ divisible by $h$, an $H$-factor of $G$ is a collection of $n / h$ copies of $H$ whose vertex sets partition $V(G)$, and each copy of $H$ is a subgraph of $G$. We say that a graph $G$ is a union of $\ell H$-factors if there exist $H_{1}, \ldots, H_{\ell}$ such that $V\left(H_{i}\right)=V(G)$ for every $i \in[\ell], E(G)=\bigcup_{i=1}^{\ell} E\left(H_{i}\right)$, and every $H_{i}$ is an $H$-factor of $G$.

We now present several theorems and observations which will be useful in our proofs, starting with the proof of Lemma 1.9 mentioned in the previous section.

Proof of Lemma 1.9. We in fact prove a stronger result. Namely, we show that there exists a bijection between pairs $\left(G, f_{G}\right)$ and $\left(\mathcal{H}, f_{\mathcal{H}}\right)$ where $G \in \mathcal{G}_{n, r}, f_{G}$ is a coloring of $G, \mathcal{H} \in \mathcal{H}_{n, r}$ and $f_{\mathcal{H}}$ is an edge coloring of $\mathcal{H}$, such that $f_{G}$ and $f_{\mathcal{H}}$ use the same number of colors and $f_{G}$ is proper if and only if $f_{\mathcal{H}}$ is proper.

We first describe a bijection between $\mathcal{G}_{n, r}$ and $\mathcal{H}_{n, r}$. Consider a graph $G \in \mathcal{G}_{n, r}$ obtained by a union of $r$ triangle factors $H_{1}, \ldots, H_{r}$, each factor containing $n$ triangles. We construct a hypergraph $\mathcal{H}$ with parts $V_{1}, \ldots, V_{r}$, each of size $n$, in the following way. For every $1 \leq i \leq r$ and for every triangle $T \in H_{i}$ we have a vertex $v_{T} \in V_{i}$. For every vertex $v \in V(G)$ we have an edge $e_{v} \in E(\mathcal{H})$ consisting of the $r$ vertices representing the $r$ triangles containing $v$. It is easy to see that the constructed hypergraph $\mathcal{H}$ is indeed a member of $\mathcal{H}_{n, r}$ and that this is a bijection (in fact, $G$ is the line graph of $\mathcal{H})$.

Given $G$ and the corresponding $\mathcal{H}$, the bijection between colorings $f_{G}$ of $G$ and edge colorings $f_{\mathcal{H}}$ of $\mathcal{H}$ is the obvious one: $f_{G}(v)=f_{\mathcal{H}}\left(e_{v}\right)$. Finally, note that $f_{G}$ is a proper coloring if and only if $f_{\mathcal{H}}$ is a proper edge coloring. Indeed, any two vertices $u, v$ in $G$ are adjacent if and only if they belong 
to the same triangle $T$ in (at least) one of the factors, which happens if and only if $v_{T} \in e_{u} \cap e_{v}$.

Observation 2.1. Let $G$ be a graph on $n+1$ vertices with chromatic number $n$. Then $G$ contains $K_{n}$ as a subgraph.

Proof. Let $V(G)=\left\{v_{1}, \ldots, v_{n+1}\right\}$ and assume for a contradiction that $G$ does not contain a copy of $K_{n}$ as a subgraph. We claim that $\bar{G}$ must contain either a triangle or a matching of size two. Indeed, otherwise all edges of $\bar{G}$ must share some vertex $v_{i} \in V(G)$. But then $G-v_{i}$ is a clique on $n$ vertices, a contradiction. Now, if $\bar{G}$ contains a triangle we can color the three vertices of the triangle with one color, and color each of the remaining $n-2$ vertices with a unique new color. In the second case, there exist two independent edges $e_{1}, e_{2} \in E(\bar{G})$. We can color the two endpoints of $e_{1}$ with one color, the two endpoints of $e_{2}$ with a second color, and finally assign a unique new color to each of the remaining $n-3$ vertices. In either case we got a proper coloring of $G$ with only $n-1$ colors, a contradiction.

Observation 2.2. Let $G$ be a graph and let $d$ be an integer such that the $d$-core of $G$ is $d$-colorable. Then $G$ is d-colorable.

Proof. Consider the following vertex deletion algorithm to obtain the $d$-core of $G$ : starting with $G_{0}:=G$, for every $i \geq 0$, if $G_{i}$ contains a vertex of degree less than $d$, we choose one such vertex arbitrarily, denote it by $v_{i}$ and let $G_{i+1}:=G_{i}-v_{i}$. The algorithm terminates with $G_{k} \subseteq G$ (for some $k$ ), when there are no more vertices of degree less than $d$. It is easy to see and well known that $G_{k}$ is the (possibly empty) $d$-core of $G$, regardless of the arbitrary choices made during the process.

Note that $d_{G_{i}}\left(v_{i}\right)<d$ for every $0 \leq i \leq k-1$ and thus any proper $d$-coloring of $G_{i+1}$ can be trivially extended to a proper $d$-coloring of $G_{i}$. Since there exists such coloring for $G_{k}$ by assumption, by greedily coloring $v_{k-1}, \ldots, v_{0}$ in this order we obtain a proper $d$-coloring of $G$.

The following are two fundamental theorems in graph theory, by König and by Brooks.

Theorem 2.3 ([28], König's theorem). Every bipartite multigraph $G$ has a proper edge-coloring with $\Delta(G)$ colors.

Theorem 2.4 ([13], Brooks' theorem). Let $G$ be a connected simple graph with $\Delta(G)=\Delta$. Then $\chi(G) \leq \Delta$ unless $G$ is a complete graph or an odd cycle, in which case $\chi(G)=\Delta+1$.

In Section 3 we consider the problem of covering complete graphs by triangle factors. The following theorem deals with such graphs, where the number of vertices is divisible by six (see [8] and page 386 of [34]).

Theorem $2.5([8])$. For $n \geq 18$, if $n \equiv 0(\bmod 6)$ and $n \notin\{36,42,48,60,66,78,84,102,132\}$, then one can cover the edges of $K_{n}$ with $n / 2$ triangle factors. On the other hand, the edges of $K_{12}$ cannot be covered by six triangle factors.

On the same topic, the following result is an immediate corollary of the work of Kirkman from 1847 [27], which was subsequently completed by Ray-Chaudhuri and Wilson [30].

Theorem 2.6. $K_{n}$ can be decomposed into triangle factors if and only if $n \equiv 3(\bmod 6)$.

For the proofs of Theorems 1.4 and 1.6, we need a more general result on decompositions of complete graphs. 
Theorem 2.7 (Theorem 1.3 in [17]). Let $H$ be a simple graph on $h$ vertices with degree sequence $d_{1}, \ldots, d_{h}$ and average degree $\bar{d}$. Then there exists a decomposition of $K_{n}$ into $H$-factors for every sufficiently large $n$ satisfying the following.

(a) $n \equiv 0(\bmod h)$;

(b) $n-1 \equiv 0(\bmod \gamma)$, where $\gamma$ is the smallest positive integer such that

$$
(\gamma, \gamma / \bar{d}) \in \operatorname{span}_{\mathbb{Z}}\left\{\left(d_{i}, 1\right) \mid i \in[h]\right\} .
$$

In Section 3 we study graphs whose chromatic number and maximum degree are very close. As part of our proof we rely on the work of Molloy and Reed in [29]. In particular, we use the following.

Definition 2.8. $k_{\Delta}$ is the maximum integer $k$ such that $(k+1)(k+2) \leq \Delta$.

Definition 2.9. A c-reducer $R=(C, S)$ of a graph $G$ consists of a clique $C$ on $c-1$ vertices and a stable set $S$ such that every vertex of $C$ is adjacent to all the vertices of $S$ but none of $V(G) \backslash(S \cup C)$.

Theorem 2.10 (Theorem 5 in [29]). There is an absolute constant $\Delta_{1}$ such that for any $\Delta \geq \Delta_{1}$ and $c \geq \Delta-k_{\Delta}$, if $G$ is a graph with maximum degree at most $\Delta, \chi(G)=c+1$, and either

(i) $c \geq \Delta-k_{\Delta}+1$, or

(ii) G has no c-reducer,

then there is some vertex $v \in V(G)$ such that the subgraph induced by $\{v\} \cup N(v)$ has chromatic number $c+1$.

In the same paper, Molloy and Reed conjectured that in fact there is no need for the condition $\Delta \geq \Delta_{1}$ in Theorem 2.10.

Conjecture 2.11 (Conjecture 6 in [29]). Theorem 2.10 holds for every $\Delta$, that is, one can take $\Delta_{1}=1$.

Another paper that deals with similar topics is that of Borodin and Kostochka [12], in which they conjectured the following.

Conjecture 2.12 ([12]). Let $G$ be a graph with $\Delta(G) \geq 9$ and $\omega(G)<\Delta(G)$. Then $\chi(G)<\Delta(G)$.

Although this conjecture remains unproven, Reed [32] proved it for sufficiently large $n$.

Theorem 2.13 (Theorem 4 in [32]). There is a constant $\Delta_{2}$ such that if $G$ is a graph with $\Delta(G) \geq \Delta_{2}$ and $\omega(G)<\Delta(G)$, then $\chi(G)<\Delta(G)$. Furthermore, $\Delta_{2} \leq 10^{14}$.

In our proofs we can use either Theorem 2.10 or Theorem 2.13. Since they both involve a large lower bound on the maximum degree in graphs, we use implicitly the one with the lower such bound. To this end, we now define the integer $\Delta_{0}$ that appeared in the definition of $\mathcal{A}_{2}$.

Definition 2.14. Let $\Delta_{0}=\min \left\{\left\lceil\Delta_{1} / 2\right\rceil,\left\lceil\Delta_{2} / 2\right\rceil\right\}$, where $\Delta_{1}$ and $\Delta_{2}$ are as defined in Theorems 2.10 and 2.13, respectively.

Remark 2.15. Note that if either Conjecture 2.11 or Conjecture 2.12 is affirmed, then it follows that $\mathcal{F}_{6}$ is $k$-nice for every $k \equiv 2(\bmod 3)$. In this case, only 11 values of $k$ (all of them divisible by three) will remain not covered by Theorem 1.3. Indeed, the affirmation of Conjecture 2.11 will simply mean that $\Delta_{0}=1$, and for Conjecture 2.12 we will have that $\Delta_{0}=5$. In either case, by definition we will get $\mathcal{A}_{2}=\{n \in \mathbb{N} \mid n \equiv 2(\bmod 3)\}$. 


\section{The chromatic number of the union of triangle factors}

In this section we discuss the chromatic number of graphs obtained by a union of triangle factors. It will in fact be more convenient to discuss generalized triangle factors.

Definition 3.1. A graph $G$ is called a generalized triangle factor if every connected component of $G$ is a subgraph of a triangle, i.e., a triangle, a path of length 2, an edge, or a vertex.

Let

$$
\chi_{r}:=\max \{\chi(G) \mid G \text { is a union of } r \text { generalized triangle factors on the same set of vertices }\}
$$

and

$$
\chi_{r}^{*}:=\max \{\chi(G) \mid G \text { is a union of } r \text { triangle factors on the same set of vertices }\} .
$$

Claim 3.2. $\chi_{r}=\chi_{r}^{*}$ for every integer $r$.

Proof. Clearly, $\chi_{r}^{*} \leq \chi_{r}$ as every triangle factor is also a generalized triangle factor. The other direction follows from the simple fact that every union of $r$ generalized triangle factors is a subgraph of a union of $r$ triangle factors (not necessarily on the same vertex set).

By Claim 3.2 we can shift our focus to generalized triangle factors, which from now on will be referred to simply as factors, whereas triangle factors will be referred to as proper factors. We may also assume that the graphs we discuss are connected, since we can always restrict our analysis to a connected component that has the same chromatic number as the whole graph. We separate our analysis of $\chi_{r}$ into three cases, according to the residue of $r \bmod 3$. We thus use the following notation for $i \in\{0,1,2\}$, in order to make our arguments easier to follow, we write $\chi_{r, i}$ for $\chi_{r}$ whenever $r \equiv i(\bmod 3)$. Thus, any claim about $\chi_{r, i}$ should be interpreted as a claim about $\chi_{r}$ only for those $r$ such that $r \equiv i(\bmod 3)$.

We now prove a few useful claims to be used later in the proof of Theorem 1.7.

Claim 3.3. The edges of $K_{6}$ cannot be covered by three factors.

Proof. Consider a union of three factors on six vertices. In order to cover all fifteen edges of $K_{6}$, at least one factor has to be of size at least five. Therefore, one of the components in this factor has to be a triangle, and clearly we may as well assume that the other one is also a triangle. It is easy to see that any other factor contains at most four edges not contained in the first one. All in all, we can cover at most fourteen edges.

Claim 3.4. It is impossible to cover 18 of the edges of $K_{7}$ with three factors.

Proof. Every factor on seven vertices contains at most six edges. Therefore, in order to cover 18 of the edges of $K_{7}$, all factors should be pairwise edge-disjoint and each factor should consist of two triangles and one isolated vertex. However, every two such factors have at least one common edge.

Claim 3.5. Let $K_{k}^{-}$be the complete graph on $k$ vertices, missing one edge. The edges of $K_{2 r}^{-}$cannot be covered by $r$ factors for any $r \equiv 2(\bmod 3)$ except $r=2$. 
Proof. Let $r \equiv 2(\bmod 3), r \geq 5$, be an integer and let $G$ be a graph on $2 r$ vertices which is the union of $r$ factors $H_{1}, \ldots, H_{r}$. We need to show that $G$ does not contain a copy of $K_{2 r}^{-}$as a subgraph. We show that $G \neq K_{2 r}^{-}$, and a very similar argument, whose details we omit, shows that $G \neq K_{2 r}$ as well. First note that $\left|E\left(K_{2 r}^{-}\right)\right|=r(2 r-1)-1$, and that there are exactly two vertices in $K_{2 r}^{-}$with degree $2 r-2$, while the rest of the vertices have degree $2 r-1$.

For every $1 \leq i \leq r$, let $t_{i}$ denote the number of non-triangle connected components in $H_{i}$. Since $2 r \equiv 1(\bmod 3)$, it follows that $t_{i}>0$ for every $i$. Note that the number of edges in every such component is one less than the number of its vertices. Hence, $\left|E\left(H_{i}\right)\right|=2 r-t_{i}$. If there exist $i \neq j$ for which $t_{i}, t_{j} \geq 2$, then $|E(G)| \leq 2(2 r-2)+(r-2)(2 r-1)=r(2 r-1)-2$, so $G \neq K_{2 r}^{-}$ in this case. Assume then that $t_{i}=1$ for all $i$ but at most one. Since $2 r \equiv 1(\bmod 3)$, the only non-triangle connected component in each such factor must be an isolated vertex. If some vertex $v$ is isolated in two different factors, then $d_{G}(v)=\sum_{i=1}^{r} d_{H_{i}}(v) \leq 0 \cdot 2+2 \cdot(r-2)=2 r-4$, and thus $G \neq K_{2 r}^{-}$. Otherwise, there are $r-1>2$ different vertices with degree at most $2 r-2$, and, once again, $G \neq K_{2 r}^{-}$.

Claim 3.6. Let $G=(V, E)$ be a connected graph obtained by a union of proper factors on the same set of vertices, let $A \subseteq V$ satisfy $|A| \equiv k(\bmod 3)$ for $k \in\{1,2\}$ and let $B=A \cup N_{G}(A)$. Then

(a) $\left|N_{G}(A)\right| \geq 3-k$;

(b) If $\left|N_{G}(A)\right|=3-k$, then $B=V$.

Proof. Let $H$ be a proper factor of $G$, and let $H^{\prime}=H[B]$. Note that every triangle in $H$ containing at least one vertex of $A$ is contained entirely in $H^{\prime}$, thus implying $(a)$. If $\left|N_{G}(A)\right|=3-k$ then $H^{\prime}$ is necessarily a proper factor. Since this is true for any proper factor $H$, it follows that $E(B, V \backslash B)=\emptyset$. Since, moreover, $G$ is connected, this implies $(b)$.

Claim 3.7. Let $G$ be a connected graph obtained by a union of $r \in\{3,5\}$ proper factors such that $\Delta(G)=2 r$. Then $G$ has no $(2 r-1)$-reducer.

Proof. Assume for a contradiction that $G$ contains a $(2 r-1)$-reducer $R=(C, S)$ with $|C|=2 r-2$, and note that $S=N_{G}(C)$.

For $r=3$ we have $|C|=4 \equiv 1(\bmod 3)$. It follows by Claim 3.6(a) (applied with $A=C$ ) that $|S| \geq 2$. If $|S|=2$, then $|V(G)|=6$ by Claim 3.6(b), contrary to our assumption that $\Delta(G)=6$. On the other hand, if $|S|>2$, then $C$ and 3 vertices of $S$ form a copy of $K_{7}$ minus three edges, contrary to the assertion of Claim 3.4.

Similarly, for $r=5$ we have $|C|=8 \equiv 2(\bmod 3)$, and thus Claim 3.6(a) implies that $|S| \geq 1$. If $|S|=1$, then $|V(G)|=9$ by Claim 3.6(b), contrary to our assumption that $\Delta(G)=10$. On the other hand, if $|S|>1$, then $G$ contains a copy of $K_{10}$ minus an edge, contrary to the assertion of Claim 3.5.

Before we state and prove our next claim, recall that $\Delta_{1}$ is the constant appearing in Theorem 2.10.

Claim 3.8. For $r \in\{3,5,6\}$, if $\Delta_{1} \leq 2 r$, then $\chi_{r}<2 r$.

Proof. Let $r \in\{3,5,6\}$, let $G$ be a union of $r$ factors, and assume for a contradiction that $\Delta_{1} \leq 2 r$ but $\chi(G) \geq 2 r$. By removing edges as necessary we may assume $\chi(G)=2 r$. We may also assume that all $r$ factors are proper by Claim 3.2. In order to prove the claim, we show that $G$ must contain a 
copy of $K_{2 r}$, contrary to the assertion of Claim 3.3 (for $r=3$ ), Claim 3.5 (for $r=5$ ), and Theorem 2.5 (for $r=6$ ). Indeed, if $\Delta(G)<2 r$, then $G=K_{2 r}$ by Brooks' theorem (Theorem 2.4). Assume then that $\Delta(G)=2 r$. For $r=6$ we then have $k_{\Delta}=2$, and thus condition $(i)$ of Theorem 2.10 (here $c=2 r-1$ ) holds in this case. For $r=3$ and $r=5$, it follows by Claim 3.7 that condition (ii) of Theorem 2.10 (here, again, $c=2 r-1$ ) holds. In either case, we can apply Theorem 2.10 to deduce that there exists a vertex $v \in V(G)$ for which $\chi\left(G^{\prime}\right)=2 r$, where $G^{\prime}=G\left[\{v\} \cup N_{G}(v)\right]$. Since $d_{G}(v) \leq \Delta(G)=2 r$, it follows that $\left|V\left(G^{\prime}\right)\right| \leq 2 r+1$ and thus, by Observation 2.1, there exists a clique of size $2 r$ in $G^{\prime} \subseteq G$.

We are now ready to prove Theorem 1.7. As previously noted, we partition the proof into three cases, according to the residue of the number of factors mod 3. We prove each case in a separate claim.

Claim 3.9. $\chi_{r, 1}=2 r+1$, and $\chi_{r} \leq 2 r$ for every $r \notin \mathcal{A}_{1}$.

Proof. If $G$ is a union of $r$ factors $H_{1}, \ldots, H_{r}$, then $\Delta(G) \leq 2 r$, and therefore $\chi(G) \leq 2 r+1$. For $r=1$ the upper bound is trivially achieved as $\chi\left(K_{3}\right)=3$. For every $r>1$, Brooks' theorem (Theorem 2.4) implies that $\chi(G)=2 r+1$ if and only if $G=K_{2 r+1}$. Since there are $r(2 r+1$ ) edges in $K_{2 r+1}$, and since $\left|E\left(H_{i}\right)\right| \leq|V(G)|$ for every $i$, the only way to obtain $G=K_{2 r+1}$ is if each $H_{i}$ is a proper factor, and each edge in $G$ is covered by the $H_{i}$ 's exactly once. In other words, $H_{1}, \ldots, H_{r}$ form a decomposition of $G$ into proper factors. By Theorem 2.6, this is possible if and only if $|V(G)| \equiv 3(\bmod 6)$, or, in terms of $r$, if and only if $r \equiv 1(\bmod 3)$.

Corollary 3.10. $\chi_{r, 0}=2 r$ for every $r \in \mathcal{A}_{0}$.

Proof. Claim 3.9 implies $\chi_{r, 0} \leq 2 r$. On the other hand, for every $r \in \mathcal{A}_{0}$ the edges of $K_{2 r}$ can be covered with $r$ factors by Theorem 2.5, thus the upper bound is tight in this case.

Claim 3.11. $\chi_{r, 2} \in\{2 r-1,2 r\}$ for every $r \equiv 2(\bmod 3)$, and $\chi_{r, 2}=2 r-1$ for every $r \in \mathcal{A}_{2}$.

Proof. The upper bound $\chi_{r, 2} \leq 2 r$ is an immediate corollary of Claim 3.9. Observe that $(r-1) \equiv$ 1 (mod 3). Therefore, by Claim 3.9, we can use $r-1$ of the factors to build a graph $G$ with $\chi(G)=2(r-1)+1=2 r-1$ (in fact, $G=K_{2 r-1}$ in this case). This proves the lower bound $\chi_{r, 2} \geq 2 r-1$.

We now prove the second statement of the claim and begin with the special case $r=2$. For convenience we consider only proper factors on $3 n$ vertices for arbitrary $n$ (this is allowed by Claim 3.2), and so by Lemma 1.9 we have $\chi_{2}=\max _{\mathcal{H} \in \mathcal{H}_{n, 2}} \chi^{\prime}(\mathcal{H})$. Note that for every $n$, every $\mathcal{H} \in \mathcal{H}_{n, 2}$ is in fact a bipartite (multi)graph with $\Delta(\mathcal{H})=3$. By König's theorem (Theorem 2.3), every such hypergraph satisfies $\chi^{\prime}(\mathcal{H})=3$.

Now let $r \in \mathcal{A}_{2} \backslash\{2\}$, let $G$ be a union of $r$ factors, and assume for a contradiction that $\chi(G)=2 r$. In order to complete the proof of Claim 3.11, we show that, contrary to the assertion of Claim 3.5, $G$ must contain a copy of $K_{2 r}$. Indeed, if $\Delta(G)<2 r$, then $G=K_{2 r}$ by Brooks' theorem (Theorem 2.4). Assume then that $\Delta(G)=2 r$. If $2 r \geq \Delta_{2}$ then Theorem 2.13 implies that $\omega(G) \geq \Delta(G)=2 r$. Otherwise, $2 r \geq \Delta_{1}$ by the definitions of $\Delta_{0}$ and $\mathcal{A}_{2}$. Claim 3.8 then implies that $r>5$ and so for $\Delta=2 r$ we have $k_{\Delta} \geq 2$, and thus condition $(i)$ of Theorem 2.10 (here $c=2 r-1$ ) holds. Hence, there exists a vertex $v \in V(G)$ for which $\chi\left(G^{\prime}\right)=2 r$, where $G^{\prime}=G\left[\{v\} \cup N_{G}(v)\right]$. Since $d_{G}(v) \leq 2 r$, it follows that $\left|V\left(G^{\prime}\right)\right| \leq 2 r+1$ and thus, by Observation 2.1, there exists a clique of size $2 r$ in $G^{\prime} \subseteq G$. 
Remark 3.12. Observe that in the proofs of Claim 3.9, Corollary 3.10 and Claim 3.11, the lower bound for $\chi_{r, i}$ (for $i=1,0,2$, respectively) was in fact given by a clique construction. Therefore, for every $r \in \mathcal{A}$, the edges of $K_{\chi_{r}}$ can be covered by $r$ factors.

To conclude this section, we consider two of the values of $r$ which are not covered by Theorem 1.7, namely, $r=3$ and $r=6$.

It is quite easy to cover the edges of $K_{5}$ with three factors (we omit the straightforward details), and thus it follows by Claim 3.9 that $5 \leq \chi_{3} \leq 6$. We do not know which of these two bounds is tight, but let us point out that if Conjecture 2.11 is true, then $\chi_{3}=5$ holds by Claim 3.8, thus providing an affirmative answer to Question 1.8 .

Finally, $\chi_{6} \leq 12$ by Claim 3.9. Although we know by Theorem 2.5 that the edges of $K_{12}$ cannot be covered by six proper factors, this does not imply, of course, that $\chi_{6}<12$. We give the following construction to show that $K_{11}$ can be covered by six factors, implying that $11 \leq \chi_{6} \leq 12$. Here, too, if Conjecture 2.11 is true, then $\chi_{6}=11$ holds by Claim 3.8 .

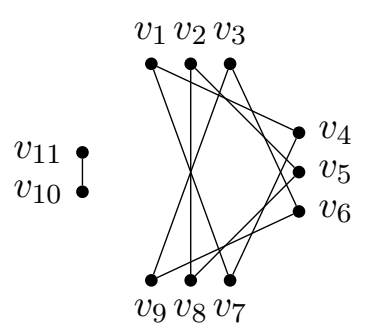

(a)

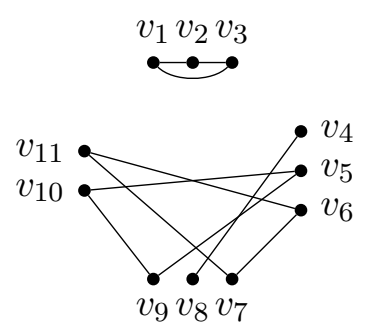

(d)

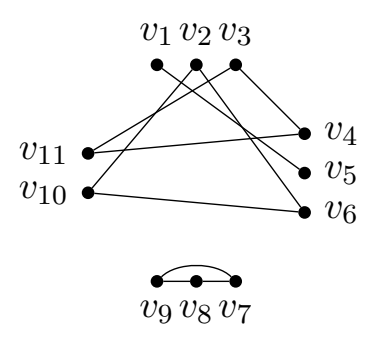

(b)

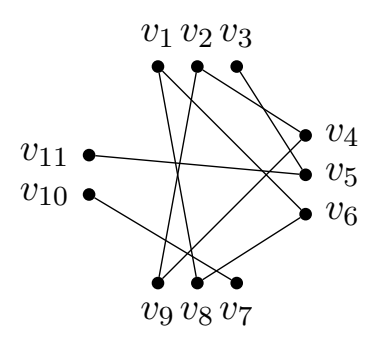

(e)

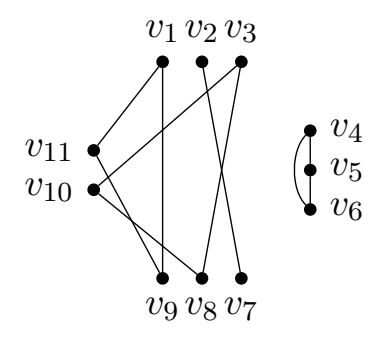

(c)

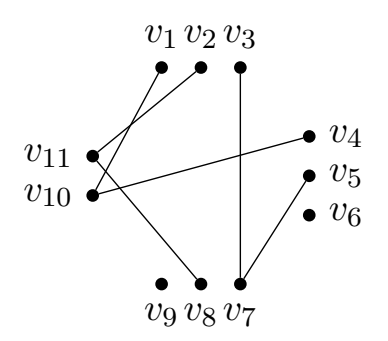

(f)

Figure 1: Covering $K_{11}$

\section{Ramsey niceness}

In this section we prove Theorems 1.3, 1.4, and 1.6. For convenience, we view niceness in a slightly different way. Let $\mathcal{F}$ be a family of fixed graphs.

Definition 4.1. Let $c_{k}(\mathcal{F})$ be the maximum integer s such that there exists a $k$-coloring of $E\left(K_{s}\right)$ with no monochromatic copy of any $F \in \mathcal{F}$.

Note that $c_{k}(\mathcal{F})=R_{k}(\mathcal{F})-1$ for every family $\mathcal{F}$ and every integer $k$.

Definition 4.2. Let $g_{k}(\mathcal{F})$ be the maximum integer s such that there exists a graph $G$ with $\chi(G)=s$ and a $k$-coloring of $E(G)$ with no monochromatic copy of any $F \in \mathcal{F}$. 
Note that a family $\mathcal{F}$ is $k$-nice if and only if $c_{k}(\mathcal{F})=g_{k}(\mathcal{F})$, and that, by definition, $c_{k}(\mathcal{F}) \leq g_{k}(\mathcal{F})$. Hence, in order to prove that a family $\mathcal{F}$ is $k$-nice, it suffices to prove that $c_{k}(\mathcal{F}) \geq g_{k}(\mathcal{F})$.

Recall our notation for families of connected graphs consisting of 3 edges, namely $\mathcal{F}_{3}=\left\{S_{3}\right\}$, $\mathcal{F}_{4}=\left\{K_{3}, P_{4}\right\}, \mathcal{F}_{5}=\left\{K_{3}, S_{3}\right\}, \mathcal{F}_{6}=\left\{P_{4}, S_{3}\right\}$, and $\mathcal{F}_{7}=\left\{K_{3}, P_{4}, S_{3}\right\}$. We prove the niceness of these families (as stated in Theorems 1.3 and 1.4) in four separate claims.

Claim 4.3. $c_{k}\left(\mathcal{F}_{6}\right)=g_{k}\left(\mathcal{F}_{6}\right)=\chi_{k}$ for every $k \in \mathcal{A}$, where $\chi_{k}$ is as defined in Section 3. In particular, $\mathcal{F}_{6}$ is $k$-nice for every $k \in \mathcal{A}$.

Proof. Let $k \in \mathcal{A}$ and let $G$ be a graph such that there exists a $k$-coloring of its edges with no monochromatic copy of any element of $\mathcal{F}_{6}$. Every color class in such a coloring must be a generalized triangle factor and thus by definition $g_{k}\left(\mathcal{F}_{6}\right)=\chi_{k}$. By Remark 3.12 we get $c_{k}\left(\mathcal{F}_{6}\right)=g_{k}\left(\mathcal{F}_{6}\right)=\chi_{k}$.

Claim 4.4. $c_{k}\left(\mathcal{F}_{3}\right)=g_{k}\left(\mathcal{F}_{3}\right)=c_{k}\left(\mathcal{F}_{5}\right)=g_{k}\left(\mathcal{F}_{5}\right)=2 k+1$ for every positive integer $k$ with the only exception $c_{1}\left(\mathcal{F}_{5}\right)=2$. In particular, $\mathcal{F}_{3}$ is $k$-nice for every $k \geq 1$ and $\mathcal{F}_{5}$ is $k$-nice for every $k \geq 2$.

Proof. The fact that $\mathcal{F}_{3}$ is $k$-nice for every $k$ follows from the result in [21] that every star is $k$-nice for every $k$. For the sake of completness, we include a simple proof. Let $G$ be a graph such that there exists a $k$-coloring of its edges with no monochromatic copy of $S_{3}$. Then the maximum degree in every color class is at most 2 . Therefore $\Delta(G) \leq 2 k$, and thus $g_{k}\left(\mathcal{F}_{3}\right), g_{k}\left(\mathcal{F}_{5}\right) \leq 2 k+1$ for every $k$. On the other hand, in 1890 Walecki showed a decomposition of the complete graph $K_{2 k+1}$ into $k$ Hamilton cycles for every $k$ (see, e.g., $[6,7]$ ). By assigning each Hamilton cycle a different color we conclude that $c_{k}\left(\mathcal{F}_{3}\right) \geq 2 k+1$ for every $k \geq 1$ and $c_{k}\left(\mathcal{F}_{5}\right) \geq 2 k+1$ for every $k \geq 2$ (note that the latter argument does not apply to $c_{1}\left(\mathcal{F}_{5}\right)$ since in this case $\left.C_{n}=K_{3} \in \mathcal{F}_{5}\right)$.

It remains to deal with $c_{1}\left(\mathcal{F}_{5}\right)$ and $g_{1}\left(\mathcal{F}_{5}\right)$. Clearly, the maximal clique containing no triangles is $K_{2}$, therefore $c_{1}\left(\mathcal{F}_{5}\right)=2$. For a general graph, $C_{5}$ is an example of an $\mathcal{F}_{5}$-free graph with chromatic number 3 , thus showing $g_{1}\left(\mathcal{F}_{5}\right) \geq 3$.

Claim 4.5. $c_{1}\left(\mathcal{F}_{4}\right)=g_{1}\left(\mathcal{F}_{4}\right)=2, c_{2}\left(\mathcal{F}_{4}\right)=g_{2}\left(\mathcal{F}_{4}\right)=3$, and $c_{k}\left(\mathcal{F}_{4}\right)=g_{k}\left(\mathcal{F}_{4}\right)=2 k-2$ for every $k \geq 3$. In particular, $\mathcal{F}_{4}$ is $k$-nice for every $k$.

Proof. Call a graph in which every connected component is a star a galaxy. Note that if an edge coloring of an arbitrary graph contains no monochromatic copy of any element of $\mathcal{F}_{4}$, then every color class in this coloring must be a galaxy.

We first deal with the cases $k=1,2$. Since every galaxy is 2-colorable, and $K_{2}$ is obviously a galaxy, it follows that $c_{1}\left(\mathcal{F}_{4}\right)=g_{1}\left(\mathcal{F}_{4}\right)=2$. The edges of $K_{3}$ can be trivially covered by two galaxies, thus $c_{2}\left(\mathcal{F}_{4}\right) \geq 3$. Now let $G$ be a union of two galaxies. We show that $G$ is 3 -colorable, thus proving $c_{2}\left(\mathcal{F}_{4}\right)=g_{2}\left(\mathcal{F}_{4}\right)=3$. By Observation 2.2 it suffices to show that the 3-core of $G$ is empty and therefore trivially 3 -colorable. Assume for a contradiction that $G$ has a non empty 3-core $K=(V, E)$ and denote $n:=|V|, e:=|E|$. For $i=1,2$, let $G_{i}$ be the intersection of the $i$ th galaxy and $K$, and note that each $G_{i}$ is a galaxy as well, since it is a subgraph of a galaxy. Let $e_{i}:=\left|E\left(G_{i}\right)\right|$ and let $s_{i}$ be the number of stars in $G_{i}$. Note that $s_{i}+e_{i}=n$ for every $i$. Since $\delta(K) \geq 3$, every vertex $v \in V$ must have degree at least 2 in at least one of the galaxies. Hence, every $v \in V$ is the center vertex of a non trivial star (i.e., a star with at least two edges) in at least one galaxy, implying that $s_{1}+s_{2} \geq n$. This in turn implies that $e \leq e_{1}+e_{2}=\left(n-s_{1}\right)+\left(n-s_{2}\right) \leq n$, and thus the average degree in $K$ is at most 2 , a contradiction. 
We now consider the general case $k \geq 3$. We show that any graph which is the union of $k \geq 3$ galaxies is $(2 k-2)$-colorable, thus proving that $g_{k}\left(\mathcal{F}_{4}\right) \leq 2 k-2$. For better readability, we show that for every $k \geq 2$, the union of $k+1$ galaxies is $2 k$-colorable.

Let $G$ be the union of $k+1$ galaxies on the same set of vertices, let $K=(V, E)$ be the $2 k$-core of $G$ and let $n=|V|, e=|E|$. By Observation 2.2 it suffices to show that $K$ is $2 k$-colorable. If $K$ is empty, then we are clearly done; assume then that it is not. As in the union of two galaxies, for every $1 \leq i \leq k+1$, let $G_{i}$ be the galaxy obtained by the intersection of $K$ and the $i$ th galaxy on $G$, let $e_{i}:=\left|E\left(G_{i}\right)\right|$ and let $s_{i}$ be the number of stars in $G_{i}$, so $s_{i}+e_{i}=n$ for every $i$.

Since $\delta(K) \geq 2 k>k+1$, once again every vertex $v \in V$ must have degree at least 2 in at least one galaxy and therefore must be the center vertex of a non trivial star in at least one galaxy. It follows that $\sum_{i=1}^{k+1} s_{i} \geq n$. Moreover, $\delta(K) \geq 2 k$ implies that $e \geq k n$. Putting it all together we get

$$
k n \leq e \leq \sum_{i=1}^{k+1} e_{i}=\sum_{i=1}^{k+1}\left(n-s_{i}\right)=(k+1) n-\sum_{i=1}^{k+1} s_{i} \leq k n .
$$

Hence, all the inequalities above are in fact equalities, and in particular we have $\sum_{i=1}^{k+1} s_{i}=n$, which implies that there are exactly $n$ stars in total, none of them is trivial, and every vertex of $K$ is the center of exactly one star. Now note that if two vertices are star centers in the same galaxy, then they are not connected by an edge in that galaxy, and not in any other galaxy (since none of them is a star center in other galaxies). Therefore, assigning the color $i$ for the star centers of the $i$ th galaxy, for every $i \in[k+1]$, yields a legal coloring of $K$ with $k+1<2 k$ colors (recall that every vertex is a star center in some galaxy). The upper bound $g_{k}\left(\mathcal{F}_{4}\right) \leq 2 k-2$ is thus established.

For the lower bound, we now show how the edges of $K_{2 k}$ can be covered with $k+1$ galaxies for every $k \geq 2$, hence $c_{k}(\mathcal{F}) \geq 2 k-2$ for every $k \geq 3$. Denote the vertices of the clique by $\left\{v_{1}, \ldots, v_{2 k}\right\}$. For every $i \in[k]$ let the $i$ th galaxy consist of the following two stars: the first center is $v_{i}$ with $v_{i+1}, \ldots, v_{i+k-1}$ as leaves, and the second center is $v_{i+k}$ with $v_{i+k+1}, \ldots, v_{i+2 k-1}$ as leaves, where all indices are calculated modulo $2 k$ (with the one exception of $v_{2 k}$ not being referred to as $v_{0}$ ). The last galaxy is the following perfect matching: $\left\{v_{i} v_{i+k} \mid i \in[k]\right\}$. See Figure 2 .

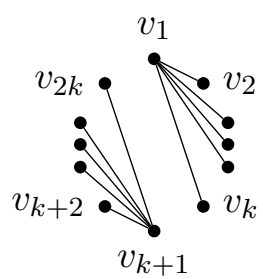

(a) The first galaxy

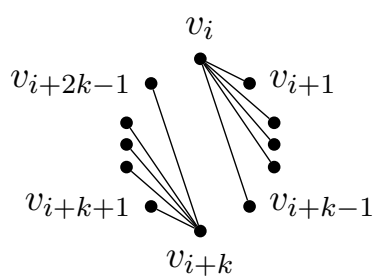

(b) The $i$ th galaxy

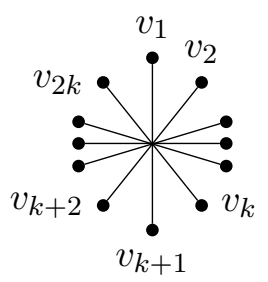

(c) The last galaxy

Figure 2: Covering $K_{2 k}$

Claim 4.6. There exists a positive integer $k_{0}$ such that $c_{k}\left(\mathcal{F}_{7}\right)=g_{k}\left(\mathcal{F}_{7}\right)=\frac{4 k}{3}+1$ for every $k \geq k_{0}$ satisfying $k \equiv 6(\bmod 9)$. In particular, $\mathcal{F}_{7}$ is $k$-nice for infinitely many values of $k$.

Proof. Note that if $G$ is a graph containing no member of $\mathcal{F}_{7}$ as a subgraph, then every connected component of $G$ is a path of length at most two. Denote such a graph by SPG (Short Paths Graph) and denote a graph obtained by a union of $k$ such graphs by $k S P G$. 
Let $s=g_{k}\left(\mathcal{F}_{7}\right)$ and let $G$ be a $k S P G$ such that $|V(G)|=n$ and $G$ is $s$-critical. In particular, the minimal degree in $G$ is at least $s-1$, hence $|E(G)| \geq \frac{n(s-1)}{2}$. However, every $S P G$ on $n$ vertices contains at most $2 n / 3$ edges, therefore $|E(G)| \leq 2 k n / 3$. Putting it together we get $s \leq \frac{4 k}{3}+1$, and in particular whenever $k=9 r+6$ (for some positive integer $r$ ) we get $s \leq 12 r+9$.

Observe that the graph $P_{3}$ has a degree sequence 2,1,1 and average degree 4/3, and the smallest integer $\gamma$ such that $(\gamma, 3 \gamma / 4) \in \operatorname{span}_{\mathbb{Z}}\{(2,1),(1,1)\}$ is 4 . By Theorem 2.7 , for every sufficiently large $n$ satisfying $n \equiv 9(\bmod 12)$, there exists a decomposition of $K_{n}$ into $P_{3}$-factors, i.e., $K_{n}$ is a $k S P G$ for those values of $n$, for $k=\frac{3(n-1)}{4}$. In other words, for any sufficiently large $k$ of the form $k=9 r+6$, $K_{12 r+9}$ is a $k S P G$, thus the proof is complete.

We conclude this section with the proof of Theorem 1.6.

Claim 4.7. Let $r$ be a positive integer, and let $\mathcal{F}$ be a family of graphs such that $K_{1, r+1} \in \mathcal{F}$, and all other $F \in \mathcal{F}$ contain at least one cycle. Then, $c_{k}(\mathcal{F})=g_{k}(\mathcal{F})=k r+1$ for infinitely many values of $k$, and, in particular, $\mathcal{F}$ is $k$-nice for those values of $k$.

Proof. It is easy to see that $g_{k}(\mathcal{F}) \leq k r+1$. Indeed, assume for contradiction that $g_{k}(\mathcal{F}) \geq k r+2$, and let $G$ be a graph such that $\chi(G)=g_{k}(\mathcal{F})$ and there exists a $k$-coloring of $E(G)$ with no monochromatic copy of any $F \in \mathcal{F}$. Then by Brooks' theorem $\Delta(G) \geq k r+1$, and thus in every $k$-coloring of $E(G)$ there must be a color class in which $r+1$ edges intersect in the same vertex, and a forbidden copy of $K_{1, r+1}$ appears.

Let $g$ be the largest girth among all members $F \in \mathcal{F} \backslash\left\{K_{1, r+1}\right\}$, and let $H$ be an $r$-regular graph with girth greater than $g$, such that $|V(H)|=h$ for some integer $h$ relatively prime to $r$ (it is well known that such graphs exist, it follows for example from the technique of Erdős and Sachs [19]). By Theorem 2.7, there exists a decomposition of $K_{n}$ into $H$-factors for every sufficiently large $n$ satisfying $n \equiv 0(\bmod h)$ and $n-1 \equiv 0(\bmod r)$ (here, in the terminology of Theorem 2.7 , as $H$ is $r$-regular, we are looking for the smallest integer $\gamma$ such that $(\gamma, \gamma / r) \in \operatorname{span}_{\mathbb{Z}}\{(r, 1)\}$, which is clearly $r$ ). By the Chinese Remainder Theorem, there exist infinitely many values of $n$ satisfying the congruences modulo $h r$, since $h$ and $r$ are relatively prime. For any such $n$, let $k=\frac{n-1}{r}$. Then $K_{k r+1}$ can be decomposed into $k H$-factors, hence $c_{k}(F) \geq k r+1$.

\section{Matchings in hypergraphs}

In this section we disprove Conjecture 1.10 in a strong sense.

Let $d$ be a positive integer and let $m=\lfloor 3 d / 2\rfloor, A=[d]$ and $B=[m] \backslash[d]$. We define an $m \times m \times m 3$ uniform, 3-partite, $d$-regular simple (containing no repeated edges) hypergraph $\mathcal{H}=(V, E)$ as follows. Let $V=X \cup Y \cup Z$, where $X=\left\{x_{1}, \ldots, x_{m}\right\}, Y=\left\{y_{1}, \ldots, y_{m}\right\}$ and $Z=\left\{z_{1}, \ldots, z_{m}\right\}$. For every $i \in A$ and every $j \in B$ we add the three hyperedges $\left\{x_{i}, y_{i}, z_{j}\right\},\left\{x_{i}, y_{j}, z_{i}\right\},\left\{x_{j}, y_{i}, z_{i}\right\}$ to $E$. If $d$ is odd we also add to $E$ all the hyperedges $\left\{x_{i}, y_{i}, z_{i}\right\}$ for every $i \in A$. Note that $\mathcal{H}$ is indeed 3-partite and $d$-regular. Let $f: E \rightarrow A$ be the function which assigns each $e \in E$ the unique index $i \in A$ such that $\left|e \cap\left\{x_{i}, y_{i}, z_{i}\right\}\right| \geq 2$. Since every two edges $e_{1}, e_{2} \in E$ such that $f\left(e_{1}\right)=f\left(e_{2}\right)$ intersect, every matching $M$ in $\mathcal{H}$ contains at most one edge from the set $f^{-1}(i)$ for every $i \in A$, implying that $|M| \leq|A|=d$.

By taking disjoint copies of $\mathcal{H}$ we obtain a $d$-regular $n \times n \times n$-partite 3-uniform hypergraph (for arbitrarily large $n$ ) such that the size of the maximum matching in the hypergraph is $d \cdot n / m=2 n / 3$ 
for even $d$, and $d \cdot n / m=\frac{2 d}{3 d-1} \cdot n$ for odd $d$, thus disproving Conjecture 1.10 for every $d \geq 4$.

In [3] it is conjectured that the edges of any $r$-uniform hypergraph with maximum degree $d$ in which every pair of edges share at most $t$ common vertices can be covered by $(t-1+1 / t+o(1)) d$ matchings, where the $o(1)$ term tends to zero as $d$ tends to infinity. If true, then, by taking $r=3$ and $t=2$, this shows that the constant $2 / 3$ above is tight for large $d$. It is also worth noting that the known results about nearly perfect matchings in regular linear hypergraphs imply that every $d$ regular 3-uniform linear hypergraph on $n$ vertices contains a nearly perfect matching. In particular, it is shown in [4], using the Rödl nibble, that any such hypergraph contains a matching covering all but at most $O\left(n \ln ^{3 / 2} d / \sqrt{d}\right)$ vertices.

In relation to the example given above, we note the following. It is easy to see that every $r$-uniform $d$ regular hypergraph on $n$ vertices contains a matching that covers at least

$$
\frac{n d}{1+(d-1) r}>\frac{n}{r}
$$

vertices. Indeed, the number of edges is $e=n d / r$, and each edge intersects at most $r(d-1)$ others, hence the line-graph, whose vertices are the edges of the hypergraph where two are adjacent if and only if they intersect, has an independent set of size at least $\frac{e}{1+r(d-1)}$, implying the above estimate.

It is known that if no two edges share more than one common vertex, and $d$ is sufficiently large as a function of $r$, then there is a nearly perfect matching (see, e.g., [4]). On the other hand, if a typical pair of intersecting edges has more than one common vertex then the typical degree of an element of the line graph is significantly less than $1+(d-1) r$, hence one could expect that the above estimate is not too close to being tight for large degrees. Surprisingly we show that this is nearly tight, even if we assume that the hypergraph is $r$-uniform, $r$-partite, has no multiple edges and is regular of arbitrarily high degree.

Claim 5.1. For every prime power $p$ and every positive integer $m$ there is an $r=p+2$ uniform, $r$-partite hypergraph, which is regular of degree $d=p^{2} m$, in which the number of vertices in each of the $r$ vertex classes is $p m$ (hence the total number of vertices is $n=p(p+2) m$ ), there are no multiple edges, and the maximum size of a matching is $m$, namely, it covers only a $1 / p=1 /(r-2)$ fraction of the vertices.

Note that by the known results about the distribution of primes this implies that for every large $r$ there is an $r$-uniform $r$-partite $d$-regular hypergraph, for arbitrarily large $d$, in which no matching covers more than a $(1+o(1)) \frac{1}{r}$ fraction of the vertices. Indeed, for every large $r$ there exists an $r^{\prime}$ such that $r-o(r) \leq r^{\prime} \leq r$ and $r^{\prime}-2$ is a prime power. We can then take the construction for $r^{\prime}$ guaranteed by Claim 5.1, replace one of the $r^{\prime}$ parts with $r-r^{\prime}+1$ copies of it, and for each edge replace the vertex from that part with all new vertices corresponding to it. We get an $r$-uniform $r$-partite hypergraph of the same regularity and with the same set of matchings. By the choice of $r^{\prime}$, we get that still only a $(1+o(1)) \frac{1}{r}$ fraction of the vertices are covered by the largest matching. This bound is tight up to the $o(1)$ term, by the argument initiating this discussion.

Proof. A projective plane of order $p$ minus a point corresponds to a $(p+1)$-uniform, $(p+1)$-partite hypergraph, with $p$ vertices in each vertex class, which is $p$ regular, has $p^{2}$ edges, and every two edges intersect. Indeed, the vertex classes are just the sets $L-x$ where $x$ is the deleted point and $L$ is any line containing it. The edges are all other lines. 
Take $m$ vertex disjoint copies $P_{1}, \ldots, P_{m}$ of the above hypergraph to get a $(p+1)$-uniform $(p+1)$ partite hypergraph with vertex classes $V_{1}, \ldots, V_{p+1}$, each of size $p m$, in which the maximum matching contains $m$ edges. Add another vertex class $V_{p+2}$ of size $p m$. Now let $H$ be the $(p+2)$-uniform $(p+2)$ partite hypergraph with vertex classes $V_{1}, \ldots V_{p+2}$ whose edges are all sets $L \cup v$ for all the pairs $(L, v)$ where $L$ is an edge of some $P_{i}$ and $v \in V_{p+2}$. It is easy to check that this hypergraph satisfies all properties in the proposition.

\section{Concluding remarks and open problems}

Question 1.1, that is, the possible conjecture that for any finite family of graphs $\mathcal{F}$ that contains at least one forest, there exists a constant $k_{0}=k_{0}(\mathcal{F})$ such that $\mathcal{F}$ is $k$-nice for all $k \geq k_{0}$, remains wide open.

In the previous sections we showed that the assertion of this question holds for any family consisting of two connected graphs, each having three edges. As mentioned in the introduction, it also holds for any family consisting of one star as well as for $\mathcal{F}=\left\{P_{4}\right\}$ [21] and for any family consisting of a single matching [9].

Here are several comments about additional families for which this assertion holds. If $K_{2} \in \mathcal{F}$ then, trivially, using the notation of Section $4, c_{k}(\mathcal{F})=g_{k}(\mathcal{F})=1$ for every $k$.

If one of the members of $\mathcal{F}$ is a path with two edges and no other member of $\mathcal{F}$ is a matching, then it is easy to see that $c_{k}(\mathcal{F})=g_{k}(\mathcal{F})=k+(k \bmod 2)$ holds for all $k$. Indeed, let $G$ be a graph satisfying $\chi(G)=g_{k}(\mathcal{F})$ and let $c$ be a $k$-coloring of $E(G)$ with no monochromatic copy of any member of $\mathcal{F}$. Clearly, any color class of $c$ is a matching, implying that the maximum degree of $G$ is at most $k$. Hence, by Brooks' theorem (Theorem 2.4), the chromatic number of $G$ is $k+1$ if and only if $G \cong K_{k+1}$ (or an odd cycle for $k=2$ ), and the desired result follows from the known values of the chromatic index of complete graphs.

If one of the members of $\mathcal{F}$ is a path with two edges and another is a matching with $r+1$ edges, then any color class is a matching of size at most $r$. It is easy to see that for large $k$ the maximum size of a complete graph that can be covered by $k$ such matchings is exactly $\max \left\{s: r k \geq\left(\begin{array}{l}s \\ 2\end{array}\right)\right\}$. Indeed, one direction is trivial. For the other, if $r k \geq\left(\begin{array}{l}s \\ 2\end{array}\right)$ and $k$ is large as a function of $r$, then the edges of $K_{s}$ can be decomposed into $k$ matchings (possibly some are empty and some are large), and it is well-known that if a graph can be decomposed into $k$ matchings it can also be decomposed into $k$ matchings of nearly equal sizes (see, e.g., [11]). Since any graph with chromatic number $s$ has at least $\left(\begin{array}{l}s \\ 2\end{array}\right)$ edges, $g_{k}(\mathcal{F})<s+1$, and thus $\mathcal{F}$ is $k$-nice for all large $k$.

If one of the members of $\mathcal{F}$ is a matching of size 2 and no other member is a star, then it is easy to verify that $c_{k}(\mathcal{F})=g_{k}(\mathcal{F})=k+1$ for all $k$, as here each color class must be a star. If $\mathcal{F}$ contains a matching of size 2 and a star with $r+1$ edges, then $c_{k}(\mathcal{F})=g_{k}(\mathcal{F})=\max \left\{s: r k \geq\left(\begin{array}{l}s \\ 2\end{array}\right)\right\}$ for all large $k$. To show this, we need the fact that, if $r k \geq\left(\begin{array}{l}s \\ 2\end{array}\right)$ and $k$ is large as a function of $r$, then the complete graph $K_{s}$ can be covered by $k$ stars, each of size at most $r$. This follows, for example, from a very special case of Gustavsson's Theorem [22]. We omit the details.

The Erdös-Sós conjecture, raised in 1962 (see, for example, [38]), asserts that every graph with average degree exceeding $r-1$, must contain as a subgraph every tree with $r$ edges. This is known to be true in many cases. If the conjecture holds for some tree $T$ with $r$ edges, then for $\mathcal{F}=\{T\}$, the average degree of a graph whose edges can be colored by $k$ colors with no monochromatic copy 
of $T$ is at most $k(r-1)$. Thus each such graph has chromatic number at most $k(r-1)+1$ (as follows by considering its $k(r-1)$-core), and equality can hold if and only if the complete graph on $k(r-1)+1$ vertices can be colored as above. By results of Ray-Chaudhuri and Wilson [31] about resolvable designs, if $s \equiv r(\bmod r(r-1))$ and $s$ is sufficiently large, then $K_{s}$ can be decomposed into subgraphs, each being the vertex disjoint union of $s / r$ cliques of size $r$. As each such subgraph cannot contain a tree on $r+1$ vertices, this shows that for all large $k \equiv 1(\bmod r), c_{k}(\mathcal{F})=g_{k}(\mathcal{F})=k(r-1)+1$ for $\mathcal{F}=\{T\}$, provided we know that the Erdös-Sós conjecture holds for $T$. The same applies, of course, to any family $\mathcal{F}$ containing such a tree $T$ as well as any additional members as long as each of them contains a connected component of size at least $r+1$. Note that this gives many additional families for which the assertion of Conjecture 1.5 holds.

It is worth noting that the condition that $k \geq k_{0}(\mathcal{F})$ in Question 1.1 is necessary. Indeed, consider, for example, a family $\mathcal{F}$ that contains two members, a star with $t+1$ edges and $K_{3}$, where $t$ is large. If $t>R_{k}\left(K_{3}\right)$, then $c_{k}(\mathcal{F})=R_{k}\left(K_{3}\right)-1$ as this is the maximum number of vertices in a complete graph whose edges can be colored by $k$ colors with no monochromatic triangle. On the other hand, $g_{k}(\mathcal{F})$ here is at least the maximum chromatic number of a triangle-free graph with maximum degree at most $k t-1$, as each such graph can be decomposed, using Vizing's Theorem, into $k$ triangle-free subgraphs, each with maximum degree at most $t$. It is well known that this maximum chromatic number is $\Theta(k t / \log (k t))$, which can be much larger than $R_{k}\left(K_{3}\right)$ for large $t$ (see, e.g., [5, 26]).

The question considered here may be generalized to oriented graphs (that is, directed graphs with no cycles of length 2). For a family of oriented graphs $\mathcal{F}$ let $p_{k}(\mathcal{F})$ denote the maximum number of vertices of a tournament whose edges can be colored by $k$ colors with no monochromatic copy of any member of $\mathcal{F}$. Let $q_{k}(\mathcal{F})$ denote the maximum possible chromatic number of an oriented graph whose edges can be colored with $k$ colors with no monochromatic copy of any member of $\mathcal{F}$. Call $\mathcal{F}$ $k$-nice if $p_{k}(\mathcal{F})=q_{k}(\mathcal{F})$. The existence of graphs of high girth and high chromatic number implies that a family that contains no oriented forest is not $k$-nice for any $k$. It is not difficult to show that the family consisting of any single directed path is $k$-nice for every $k$. This can be done using the theorem which asserts that any oriented graph of chromatic number $g$ contains a directed path with $g$ vertices, a statement proved independently by Gallai [20], Roy [33], Hasse [24] and Vitaver [35]. One may study the directed analogues of Question 1.1 and Conjecture 1.5, which are the following.

Question 6.1. Is it true that for any finite family of oriented graphs $\mathcal{F}$ that contains at least one forest, there exists a constant $k_{0}=k_{0}(\mathcal{F})$ such that $\mathcal{F}$ is $k$-nice for all $k \geq k_{0}$ ?

Question 6.2. Is it true that any finite family of oriented graphs $\mathcal{F}$ that contains at least one forest is $k$-nice for infinitely many integers $k$ ?

See [36] for (somewhat) related results.

Finally, the topics discussed in this paper suggest a generalization of Ramsey numbers: For (finite or infinite) families of graphs $\mathcal{F}_{1}, \ldots, \mathcal{F}_{k}$ let $R=R\left(\mathcal{F}_{1}, \ldots, \mathcal{F}_{k}\right)$ denote the smallest integer $R$ so that for any $k$-coloring of the edges of the complete graph $K_{R}$ there is a monochromatic copy of some member $F \in \mathcal{F}_{i}$ colored in color number $i$ for some $1 \leq i \leq k$.

The study of the number $g_{k}(\mathcal{F})$ presented in Section 4 is also interesting. As far as we know, no work has been previously done to determine the value of $g_{k}(\mathcal{F})$. It is easy to see that $g_{k}(\mathcal{F})$ is finite if and only if the family $\mathcal{F}$ contains a forest. Indeed, if the chromatic number of a graph is at least $r$, then it contains a subgraph with minimum degree at least $r-1$. Hence in any $k$-edgecoloring there is a monochromatic subgraph with average degree at least $(r-1) / k$, and hence also a 
subgraph with minimum degree at least half of that. That is, for every graph $G$ with $\chi(G)=r$ and for every $k$-coloring of $E(G)$, there exists a monochromatic subgraph with minimum degree at least $(r-1) / 2 k$. This monochromatic graph contains every forest on at most $(r-1) / 2 k$ vertices. Thus, if $n_{0}$ is the order of the smallest forest in $\mathcal{F}$, then $g_{k}(\mathcal{F}) \leq 2 k n_{0}$. The other direction follows from the well-known argument by Erdős [18] that for any two positive integers $\chi, g$ there exists a graph $G$ with $\chi(G)=\chi$ and girth greater than $g$.

For directed graphs there are early papers dealing with the analogous problem for $g_{k}(\mathcal{F})$. For instance, Chvátal proved [15] that for a directed path $P$ of length $\ell$ we have $g_{k}(P)=\ell^{k}$. See also [2] for related results.

Acknowledgement The research on this project was initiated during a joint research workshop of Tel Aviv University and the Free University of Berlin on Graph and Hypergraph Coloring Problems, held in Tel Aviv in March 2017, and supported by a GIF grant number G-1347-304.6/2016. We would like to thank both institutions for their support. We would also like to thank the anonymous referees for helpful comments.

\section{References}

[1] R. Aharoni, P. Charbit, and D. Howard, "On a generalization of the Ryser-Brualdi-Stein Conjecture." Journal of Graph Theory 78, no. 2 (2015): 143-156.

[2] N. Alon, "Monochromatic directed walks in arc-colored directed graphs." Acta Mathematica Hungarica 49, no. 1-2 (1987): 163-167.

[3] N. Alon and J. H. Kim, "On the degree, size, and chromatic index of a uniform hypergraph." Journal of Combinatorial Theory, Series A 77, no. 1 (1997): 165-170.

[4] N. Alon, J. H. Kim and J. Spencer, "Nearly perfect matchings in regular simple hypergraphs." Israel Journal of Mathematics 100, no. 1 (1997): 171-187.

[5] N. Alon and J. H. Spencer, The Probabilistic Method, Fourth Edition, Wiley (2016).

[6] B. Alspach, "The wonderful Walecki construction." Bull. Inst. Combin. Appl 52 (2008): 7-20.

[7] B. Alspach, J. C. Bermond and D. Sotteau, "Decomposition into cycles I: Hamilton decompositions." in: Cycles and Rays, Springer, Dordrecht (1990): 918.

[8] A. Assaf, E. Mendelsohn, and D. R. Stinson, "On resolvable coverings of pairs by triples." Utilitas Mathematica 32 (1987): 67-74.

[9] A. Bialostocki, and A. Gyárfás, "Replacing the host $K_{n}$ by $n$-chromatic graphs in Ramsey-type results." arXiv:1506.04495 (2015).

[10] J. Bierbrauer, "Ramsey numbers for the path with three edges." European Journal of Combinatorics 7, no. 3 (1986): 205-206.

[11] J. A. Bondy, and U. S. R. Murty, Graph Theory with Applications, Elsevier, New York (1976). 
[12] O. V. Borodin, and A. V. Kostochka, "On an upper bound of a graph's chromatic number, depending on the graph's degree and density." Journal of Combinatorial Theory, Series B 23, no. 2-3 (1977): 247-250.

[13] R. L. Brooks, "On colouring the nodes of a network." in: Classic Papers in Combinatorics, Birkhuser, Boston (2009): 118-121.

[14] N. J. Cavenagh, J. Kuhl, and I. M. Wanless, "Longest partial transversals in plexes." Annals of Combinatorics 18, no. 3 (2014): 419-428.

[15] V. Chvátal, "Monochromatic paths in edge-colored graphs." Journal of Combinatorial Theory, Series B 13, no. 1 (1972): 69-70.

[16] E. J. Cockayne, and P. J. Lorimer, "The Ramsey number for stripes." Journal of the Australian Mathematical Society 19, no. 2 (1975): 252-256.

[17] P. Dukes and A. C. H. Ling, "Asymptotic existence of resolvable graph designs." Canadian Mathematical Bulletin 50, no. 4 (2007): 504-518.

[18] P. Erdős, "Graph theory and probability." Canad. J. Math 11, no. 11 (1959): 34-38.

[19] P. Erdős and H. Sachs, "Reguläre Graphen gegebener Taillenweite mit minimaler Knotenzahl." Wiss. Z. Martin-Luther-Univ. Halle-Wittenberg Math.-Natur. Reihe 12 (1963): 251-257. (in German)

[20] T. Gallai, "On directed paths and circuits." Theory of Graphs (1968): 115-118.

[21] P. Garrison, "Good graph hunting." arXiv:1508.01833 (2015).

[22] T. Gustavsson, "Decompositions of large graphs and digraphs with high minimum degree", Doctoral Dissertation, Dept. of Mathematics, Univ. of Stockholm (1991).

[23] A. Gyárfás, private communication.

[24] M. Hasse, "Zur algebraischen Begründung der Graphentheorie I." Mathematische Nachrichten 28, no. 56 (1965): 275-290. (in German)

[25] R. W. Irving, "Generalised Ramsey numbers for small graphs." Discrete Mathematics 9, no. 3 (1974): 251-264.

[26] A. Johansson, "Asymptotic choice number for triangle free graphs." DIMACS Technical Report (1996).

[27] T. P. Kirkman, "On a problem in combinations." Cambridge and Dublin Mathematical Journal 2 (1847): 191-204.

[28] D. Kőnig, "Graphok és alkalmazásuk a determinánsok és a halmazok elméletére." Mathematikai és Természettudományi Ertesito 34 (1916): 104-119. (in Hungarian)

[29] M. Molloy, and B. Reed, "Colouring graphs when the number of colours is almost the maximum degree." Journal of Combinatorial Theory, Series B 109 (2014): 134-195. 
[30] D. K. Ray-Chaudhuri and R. M. Wilson, "Solution of Kirkman's schoolgirl problem." in: Proc. Symp. Pure Math, vol. 19, (1971): 187-203.

[31] D. K. Ray-Chaudhuri and R. M. Wilson, "The existence of resolvable block designs." in: A Survey of Combinatorial Theory, North-Holland, Amsterdam (1973): 361-375.

[32] B. Reed, "A strengthening of Brooks' Theorem." Journal of Combinatorial Theory, Series B 76, no. 2 (1999): 136-149.

[33] B. Roy, "Nombre chromatique et plus longs chemins d'un graphe." Revue française d'informatique et de recherche opérationnelle 1, no. 5 (1967): 129-132. (in French)

[34] D. R. Stinson, "A survey of Kirkman triple systems and related designs." Discrete Mathematics 92, no. 1-3 (1991): 371-393.

[35] L. M. Vitaver, "Determination of minimal coloring of vertices of a graph by means of boolean powers of the incidence matrix." In Dokl. Akad. Nauk SSSR, vol. 147, no. 758-759, p. 728 (1962). (in Russian)

[36] R. Yuster, "A Ramsey type result for oriented trees." European Journal of Combinatorics 60 (2017): 1-9.

[37] D. B. West, Introduction to Graph Theory, Prentice Hall (2001).

[38] M. Woźniak, "On the Erdős-Sós Conjecture." J. Graph Theory 21, no. 2 (1996): 229-234. 